\title{
A SAMPLING MODEL TO ASCERTAIN AUTOMATION-INDUCED COMPLACENCY IN MULTI-TASK ENVIRONMENTS
}

\author{
Nasrine Bagheri \& Greg A. Jamieson \\ Cognitive Engineering Laboratory, University of Toronto, Ontario, CANADA
}

\begin{abstract}
This article discusses the development of a model that defines the optimal sampling behaviour of operators in a multi-task flight simulation, where one of the tasks is automated. The goal of this model is to assign a cost function to the attention allocation strategy of participants, allowing us to assess the efficiency of their overall strategy. The model revealed that the optimal sampling strategy should be the same regardless of the automation reliability. When applied to previously reported empirical data, the model revealed that participants using constant, highly reliable automation demonstrated more 'expensive' monitoring behaviour, However, their monitoring behaviour became more efficient over time, which is inconsistent with the conclusion that the poor overall monitoring performance was due to complacency. This model allowed us to define an optimal monitoring performance, which is an important step in being able to accurately assess "complacency".
\end{abstract}

Key words: Human-automation interaction, complacency, sampling strategy

\section{INTRODUCTION}

Operators of complex systems are often involved in multi-task environments where several information displays compete for their attention. One of the primary cognitive tasks of operators in such systems is managing their allocation of attention so that the displays are sampled at appropriate frequencies to ensure accurate system state identification (Moray \& Inagaki, 1999). The attention allocation strategy of operators can have a large influence on the safety and the efficacy of man-machine systems. 
There is a wide spectrum of tasks that operators can be asked to perform (e.g., continuous control, discrete control, monitoring, etc.) (Schumacher \& Geiser, 1983). As a result of the increased use of automation, monitoring tasks have become prevalent in modern man-machine systems. In this context, an operator's role is to monitor displays to detect abnormal states of the automated system. The high reliability of modern automated systems means that such abnormal states occur only rarely, and it has been suggested that operators may become "complacent" in their monitoring (Wiener, 1981). Complacency has been defined as "self-satisfaction which may result in non-vigilance based on an unjustified assumption of satisfactory system state" (Parasuraman et al., 1993, quoting NASA definition).

Although complacency is considered to be a serious problem, little consensus exists as to how it can be measured (Prinzel et al., 2001). Previous research has concluded that operators were complacent based primarily on their automation failure detection performance over time (e.g., Parasuraman et al., 1993, Singh et al., 1997). Moray (2000, 2003) questioned whether such evidence adequately supports the presence of "complacency" as even optimal behaviour can result in missed signals. Complacency implies undersampling, not missed signals. This emphasizes the need to assess operators' attention allocation strategies while addressing the issue of complacency.

Bagheri and Jamieson (2004) replicated a Parasuraman et al. (1993) study in which participants who interacted with a consistent and highly reliable automated system were said to show signs of complacency based primarily on detection performance. In both studies, automation reliability was varied as a between-subject factor. The reliability was either constant at a high level $(87.5 \%)$, constant at a low level $(56.25 \%)$, or changed every 10 minutes from high $(87.5 \%)$ to low $(56.25 \%)$. In addition to detection performance, Bagheri \& Jamieson (2004) recorded participants' eye movements to determine whether attention allocation corroborated the conclusions reached by Parasuraman et al. (1993). Results confirmed the significant effect of automation reliability on detection rate; participants using automation of constant and high reliability had the poorest performance. Eye movements revealed that these participants sampled the monitoring task significantly less than participants in the constant low and variable reliability conditions. However, the evolution of their attention allocation patterns did not appear to support the attribution of their poor performance to complacency.

The observational data collected by Bagheri \& Jamieson (2004) did not afford a conclusion as to whether participants' sampling behaviour was adequate. To reach such a conclusion, a formal method of evaluating participants' attention allocation strategy is required. Moray and Inagaki (2000) showed that the occurrence of complacency cannot be proved unless an optimal behaviour is specified as a benchmark. This article presents a 
model that defines the optimal sampling behaviour based on the characteristics of the tasks that participants had to perform in the study with the goal of concluding whether participants showed 'complacent', 'eutectic' or 'sceptical' monitoring behaviour (Moray, 2003). The eye movement data collected by Bagheri and Jamieson (2004) are reanalyzed to determine which automation reliability condition leads to more effective performance.

\section{METHOD}

\subsection{Apparatus}

We present here a model of sampling behaviour for interaction with the Multi-Attribute Task battery used in Parasuraman et al. (1993) (Figure 1). The MAT Battery is a flight simulation that requires participants to perform three equally important tasks: (1) automated system-monitoring, (2) manual tracking, and (3) manual fuel management. The windows containing the information required to perform each task were defined as lookzones.

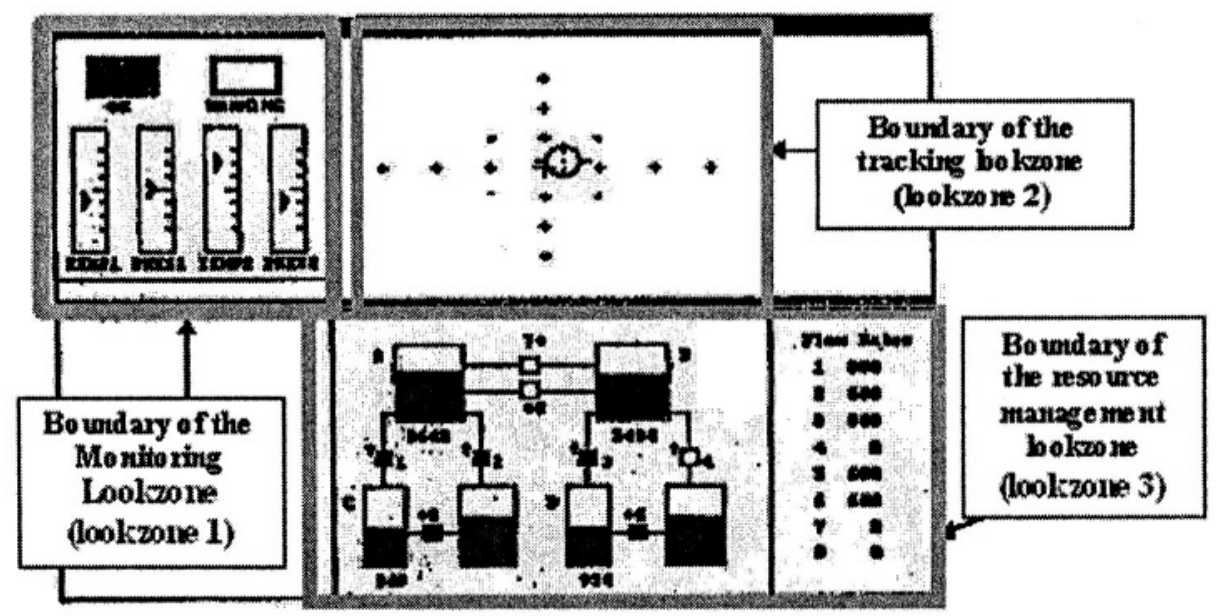

Figure 1. Picture of the MAT battery with the three lookzones of interest highlighted.

The system-monitoring task (lookzone 1) consisted of four engine gauges that participants had to monitor for randomly occurring abnormal values that represented system malfunctions. The monitoring task was automated so that a gauge showing an abnormal value would normally reset itself without participant intervention. Participants were advised that the automated system would sometimes fail to correct these malfunctions. Automation reliability 
was defined as the percentage of malfunctions corrected by the automation. In case of an automation failure, participants were required to correct the malfunction manually. If they did not detect the failure within 10 seconds, the pointer was automatically reset and the event was scored as a "miss". Participants were not informed that they missed a failure.

The goal of the tracking task (lookzone 2) was to keep the aircraft within a rectangular area using a joystick (first-order control). The goal of the fuel management task (lookzone 3) was to keep the fuel level in tanks A and B between 2000 and 3000 gallons by controlling a network of eight pumps.

An Eye-gaze Response Interface Computer Aid (ERICA) system was also used to track participants' eye movements in the lookzones defined earlier. The eye-tracker used infrared light technology and was non-invasive to participants. Gaze location samples were taken 30 times per second.

\subsection{Model of attention}

Our goal was to develop a normative optimal sampling model for interacting with the MAT battery. As participants were advised that the tasks were of equal importance, the model would define the behaviour to optimize performance on the three tasks simultaneously. Our goal was to define a model to assess attention allocation strategy globally, as opposed to a local optimization approach wherein each eye movement is considered as a decision that aims at maximizing the marginal utility of the next fixation. For that purpose, we assumed that a functional relationship could be established between participants' average performance and their sampling strategy.

\subsubsection{Definition of the problem constraints}

There were three lookzones of interest corresponding to the three tasks performed by participants. Let $x, y$, and $z$ be the sampling rates of the monitoring, tracking, and resource management lookzone, respectively. As a conservative practical approximation, we assumed that only one source of information can be monitored at a time (Moray and Inagaki, 1999). $0.5 \mathrm{sec}$ was established as a lower bound on dwell time in "real-life" tasks for experimented operators (Moray, 1986). Since participants had never used the MAT battery, we assumed a dwell time of $1 \mathrm{sec}$, and thus an eye movement rate less than or equal to 1 fixation per second. This constraint is expressed below:

$$
x+y+z \leq 1
$$


In this model, each lookzone is allocated a specific elementary cost function which depends only on the associated sampling rate. Hence, we defined $C_{m}(x), C_{t}(y), C_{r}(z)$ as the elementary cost function associated with the monitoring, tracking, and the resource task, respectively. The overall cost is the sum of the contributions of these three independent elementary costs:

$$
\Omega(x, y, z)=C_{m}(x)+C_{t}(y)+C_{r}(z)
$$

\subsubsection{Definition of the cost function requirements}

The elementary cost functions must meet three requirements. First, as the three tasks are equally important, missing all of the automation failures must have the same cost as always keeping the aircraft outside the rectangular area, which in turn must have the same cost as keeping the fuel level always beyond the limit. This requirement is expressed as follows:

$$
\text { Requirement (1): } \quad C_{m}(0)=C_{t}(0)=C_{r}(0)
$$

The second requirement implies that detecting all automation failures should be as rewarded as always keeping the aircraft within the rectangular area, or keeping the fuel level within limits at all times. Assuming that given the greatest possible sampling rate of a given lookzone, participants are sure to perform the task perfectly, this constraint is expressed as:

$$
\text { Requirement (2): } \quad C_{m}(1)=C_{t}(1)=C_{r}(1)
$$

Let $\boldsymbol{C}(\boldsymbol{\eta})$ represent any of the three elementary cost functions. $\boldsymbol{C}(\boldsymbol{\eta})$ has a direct physical meaning if Requirements (1) and (2) are translated into $\mathrm{C}(0)$ $=1$ and $\mathrm{C}(\mathrm{l})=0$. It represents the percentage of what is missed over what must be achieved for the performance on a given task to be considered perfect. Thus, $C_{m}(x)$ is the percentage of automation failures undetected, $C_{I}(y)$ the percentage of time the aircraft spends outside the rectangular area, and $C_{r}(z)$ the percentage of time the fuel level is outside the targeted limit.

Another important requirement is that the elementary cost functions be decreasing. Indeed, the higher the sampling rate, the better the participant performs in the lookzone and the lower the corresponding cost. Hence the fundamental requirements for each of the elementary cost functions are:

Requirement (1): $\mathrm{C}(0)=1$

Requirement (2): $\mathrm{C}(1)=0$

Requirement $(3): C^{\prime}(\eta) \leq 0$ for $\eta \in[0 ; 1]$ 
Two additional considerations were made to find the most realistic elementary cost functions. First, when the sampling rate is very low (close to 0 ), participants are expected to perform poorly. The elementary cost would thus remain very close to 1 for any sufficiently small values of $\eta$. This implies that the slope be more or less horizontal at $\eta=0$ (i.e. $C^{\prime}(0) \approx 0$ ).

Similarly, when the sampling rate is high enough, participants perform almost perfectly in the lookzone regardless of how close to 1 the sampling rate actually is. This implies $C^{\prime}(1) \approx 0$ (i.e., the slope at $\eta=0$ almost null).

\subsubsection{Definition of the elementary cost functions and their parameters.}

A continuous mathematical expression of $C(\eta)$ was needed for the optimization process of $\Omega(x, y, z)$. Given the requirements and considerations stated above, we made the assumption that $C(\eta)$ had the following form:

$$
C(\eta)=1-\frac{1-e^{-\alpha \eta^{\beta}}}{1-e^{-\alpha}}
$$

where $\alpha$ and $\beta$ have to be determined for each of the three lookzones.

Equation (1) satisfies Requirements (1), (2) and (3) automatically. For a given lookzone, parameters $\alpha$ and $\boldsymbol{\beta}$ were calculated so as to make $\boldsymbol{C}(\boldsymbol{\eta})$ defined by Equation (1) fit a series of experimental data. To do so, three elementary costs, $d, e$, and $f$ associated with three sampling rates, $a, b$, and $c$ (arbitrarily chosen, and such that $a<b<c$ ) were experimentally determined. Given a sampling rate $\eta$, an estimate of the $\operatorname{cost} C(\eta)$ can be obtained by averaging the percentage of what is missed in the zone over several test sessions when the operator looks at it for one second every $1 / \eta$ seconds (periodic sampling). Given the experimental points $(a, d),(b, e)$ and $(c, f)$, we defined the slope parameter: $\mu=(f-d) /(c-a)$ (Figure 2).

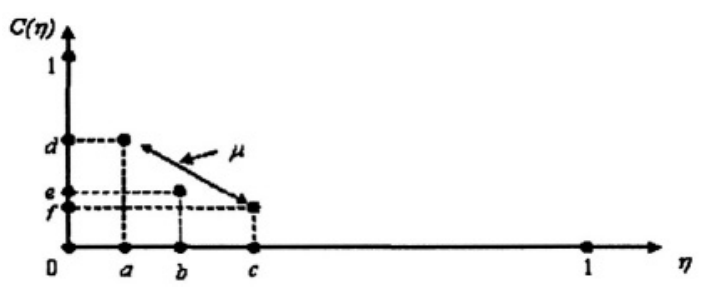

Figure 2. Construction of the elementary cost function based on three data points

$\boldsymbol{\alpha}$ and $\boldsymbol{\beta}$ were determined to make $C(\boldsymbol{\eta})$ fit these three points as closely as possible using the following technique. First, we imposed that the actual 
value of the cost function at the sampling rate $b$ (middle point) be equal to its experimental estimation, $e$. Then, we assumed that the slope at this point could be approximated by $\boldsymbol{\mu}$. Hence, we solved for $\boldsymbol{\alpha}$ and $\boldsymbol{\beta}$ in order to get:

(2)

$$
C(b)=e
$$

\section{Equation}

$C^{\prime}(b)=\mu$

Equation (3)

Using Equations (1) and (2), $\boldsymbol{\beta}$ can be expressed in terms of $\boldsymbol{\alpha}, b$, and $e$ :

$$
\beta=\frac{1}{\ln b} \cdot \ln \mid-\frac{1}{\alpha} \ln (1-(1-e)(1-\exp (-\alpha)) \mid
$$

Plugging this last expression into Equation(3), $\boldsymbol{\alpha}$ solves the equation below:

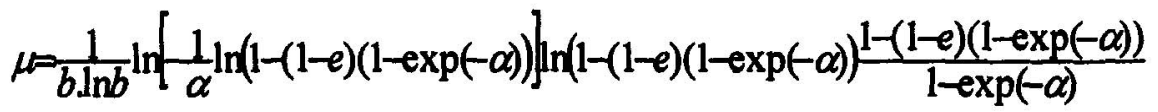

Finally, a validation criterion was established to verify that the computed cost function $\boldsymbol{C}(\boldsymbol{\eta})$ gave a fairly good representation of the problem. The cost estimation at a given sampling rate may greatly fluctuate from one test session to another. Given the sampling rate $\boldsymbol{\eta}$, the radius $\boldsymbol{R}(\boldsymbol{\eta})$ was defined as the greatest deviation to the mean value of cost estimate over the performed test sessions. Then, using Equation (1) for $\boldsymbol{\eta}=\boldsymbol{a}$ and $\boldsymbol{\eta}=\boldsymbol{c}$, we stated that:

\section{If $C(a) \in[d-R(d) ; d+R(d)]$ and if $C(c) \in[f-R(f) ; f+R(f)]$,}

Then, $C(\eta)$ given by Equation (1) was a sufficiently good representation.

Otherwise, the process had to be repeated and $\boldsymbol{\alpha}$ and $\boldsymbol{\beta}$ had to be redefined, using other arbitrary sampling rates $a, b$, and $c$. The criterion states that $C(a)$ and $C(c)$ must remain within the acceptable range of variations about the estimated mean value of the cost at $\boldsymbol{\eta}=\boldsymbol{a}$ and $\boldsymbol{\eta}=\boldsymbol{c}$, respectively $d$ and $f$.

\subsubsection{Experimental determination of the cost functions' parameters}

The next step in the model was to determine experimentally $\boldsymbol{\alpha}$ and $\boldsymbol{\beta}$ for the three elementary cost functions. A pilot study was conducted where participants were asked to sample periodically one particular lookzone at a given rate. A metronome was used to pace the sampling. The performance cost associated with a given sampling rate was evaluated.

Cost associated with the monitoring task. The percentage of automation failures missed while periodically sampling the monitoring lookzone every $1 / x$ seconds was determined. $1 / x$ tests were performed corresponding to the different possible times the operator could start to sample in the $[0 ; 1 / x \mathrm{sec}]$ 
different possible times the operator could start to sample in the $[0 ; 1 / x \mathrm{sec}]$ starting interval. The detection rate was then averaged over the number of tests. The three following sampling rates were chosen for both the high and the low reliability conditions: $a=1 / 28 \mathrm{~s}^{-1}, b=1 / 20 \mathrm{~s}^{-1}$, and $c=1 / 14 \mathrm{~s}^{-1}$. For the high reliability case, we found $\alpha=85.08$ and $\beta=1.57$. Hence:

$$
C_{m}(x)=1-\frac{1-e^{-85.08 \times 1.57}}{1-e^{-85.08}}
$$

For the low reliability case, we determined $\alpha=97.52$ and $\beta=1.63$. Hence:

$$
C_{m}(x)=1-\frac{1-e^{-97.52 x^{1.63}}}{1-e^{-97.52}}
$$

Table 1. Experimental and analytical values of the monitoring cost function by sampling rate for the high reliability condition (87.5\%) and for the low reliability condition $(56.25 \%)$.

\begin{tabular}{|c|c|c|c|c|}
\cline { 2 - 5 } \multicolumn{1}{c|}{} & \multicolumn{3}{c|}{ Cost (\% missed automation failures) } \\
\cline { 2 - 5 } \multicolumn{1}{c|}{$\begin{array}{c}\text { Sampling } \\
\text { rate }(x)\end{array}$} & $\begin{array}{c}\text { Experimentan } \text { (migh (87.5\%) } \\
\text { (mean value } \pm \text { radius }\end{array}$ & $\begin{array}{c}\text { Analytical } \\
\text { value } C_{m}(x)\end{array}$ & $\begin{array}{c}\text { Experimental estimation } \\
\text { (mean value } \pm \text { radius) }\end{array}$ & $\begin{array}{c}\text { Analytical } \\
\text { value } C_{m}(x)\end{array}$ \\
\hline $1 / 14$ & $32 \% \pm 7 \%$ & $26 \%$ & $26 \% \pm 3 \%$ & $27 \%$ \\
$1 / 20$ & $46 \% \pm 4 \%$ & $46 \%$ & $48 \% \pm 3 \%$ & $48 \%$ \\
$1 / 28$ & $72 \% \pm 11 \%$ & $63 \%$ & $67 \% \pm 2 \%$ & $65 \%$ \\
\hline
\end{tabular}

Cost associated with the tracking task. The cost of the tracking task was estimated by calculating the percentage of time spent outside the rectangular area for a given periodic sampling rate. The following sampling rates were tested: $a=1 / 10 \mathrm{~s}^{-1}, b=1 / 8 \mathrm{~s}^{-1}$, and $c=1 / 5 \mathrm{~s}^{-1}$. For each of them, a five-minute session was performed by five different operators. The corresponding costs were then averaged, and led to $\alpha=10.86$ and $\beta=1.04$. Hence:

$$
C_{1}(y)=1-\frac{1-e^{-10.86 y^{1.04}}}{1-e^{-10.86}}
$$

Table 2. Experimental and analytical values of the tracking cost function by sampling rate

\begin{tabular}{|c|c|c|}
\cline { 2 - 3 } \multicolumn{1}{c|}{} & \multicolumn{2}{c|}{ Cost (\% time outside of the rectangular area) } \\
\hline Sampling rate $(y)$ & $\begin{array}{c}\text { Experimental estimation } \\
\text { (mean value } \pm \text { radius) }\end{array}$ & $\begin{array}{c}\text { Analytical value } \\
C_{t}(y)\end{array}$ \\
\hline $1 / 5$ & $11 \% \pm 2 \%$ & $13 \%$ \\
$1 / 8$ & $29 \% \pm 5 \%$ & $29 \%$ \\
$1 / 10$ & $41 \% \pm 6 \%$ & $37 \%$ \\
\hline
\end{tabular}


Cost associated with the resource management task. The cost of the resource management task was established by evaluating the percentage of time the fuel level was beyond the targeted limit when the lookzone was periodically sampled at a given rate. This task greatly differed from the other two as the performance largely depends on the strategy of the operator. This led to large fluctuations around the mean value when trying to evaluate the cost. The following sampling rates were chosen: $a=1 / 180 \mathrm{~s}^{-1}, b=1 / 120 \mathrm{~s}^{-1}$, and $c=1 / 60 \mathrm{~s}^{-1}$. For each, three different operators performed 4 ten-minute blocks. Costs were then averaged and led to: $\alpha=\mathbf{5 2 . 4 3}$ and $\beta=\mathbf{0 . 8 4}$. Hence:

$$
C r(z)=1-\frac{1-e^{-52.43 z^{0.84}}}{1-e^{-52.43}}
$$

Table 3. Experimental and analytical values of the resource cost function by sampling rate

\begin{tabular}{|c|c|c|}
\hline \multirow{2}{*}{ Sampling rate $(z)$} & \multicolumn{2}{c|}{ Cost (\% time outside of the targeted limit) } \\
& $\begin{array}{c}\text { Experimental estimation } \\
\text { (mean value } \pm \text { radius) }\end{array}$ & $\begin{array}{c}\text { Analytical value } \\
C_{r}(z)\end{array}$ \\
\hline $1 / 60$ & $18 \% \pm 12 \%$ & $19 \%$ \\
$1 / 120$ & $39 \% \pm 22 \%$ & $39 \%$ \\
$1 / 180$ & $59 \% \pm 31 \%$ & $51 \%$ \\
\hline
\end{tabular}

\subsubsection{Model verification}

In order to verify that the monitoring cost function generated was sufficiently realistic, it was compared to additional experimental data. Table 4 summarizes the estimated monitoring cost for both reliability conditions and compares these experimental data to the analytical values obtained using the monitoring cost functions defined earlier. We can thus see that data obtained from the model are very close to those obtained experimentally.

Table 4. Percentage of automation failures missed by reliability and sampling rate determined experimentally and using the model

\begin{tabular}{|l|l|l|l|l|}
\cline { 2 - 5 } \multicolumn{1}{c|}{} & \multicolumn{4}{c|}{ Estimated percentage of failures missed } \\
\cline { 2 - 5 } \multicolumn{1}{c|}{ Hampling rate } & From the model & Experimental & From the model & Experimental \\
\hline $1 / 5 \mathrm{~s}^{-1}$ & 0.1 & 0 & 0.08 & 0 \\
$1 / 15 \mathrm{~s}^{-1}$ & 29.8 & 33.33 & 30.7 & 34.76 \\
$1 / 20 \mathrm{~s}^{-1}$ & 46.2 & 46.33 & 47.8 & 48.4 \\
$1 / 50 \mathrm{~s}^{-1}$ & 83.3 & 79.83 & 84.7 & 80.31 \\
$1 / 100 \mathrm{~s}^{-1}$ & 94 & 89.92 & 94.8 & 90.2 \\
$1 / 200 \mathrm{~s}^{-1}$ & 97.9 & 94.96 & 98.3 & 95.52 \\
\hline
\end{tabular}




\subsubsection{Problem formulation and resolution}

The optimal attention allocation strategy is given by the minimization of the overall cost function $\Omega(x, y, z)$ on the domain that satisfies the constraint: $x+y+z \leq 1$, where $x, y$ and $z$ are comprised between 0 and 1 .

1. For the blocks with high reliability automation:

$$
\Omega(x, y, z)=3-\frac{1-e^{-85.08 x^{1.57}}}{1-e^{-85.08}} \cdot \frac{1-e^{-10.86 y^{1.04}}}{1-e^{-10.86}}-\frac{1-e^{-52.43 z^{0.84}}}{1-e^{-52.43}}
$$

2. For the blocks with low reliability automation:

$$
\Omega(x, y, z)=3-\frac{1-e^{-97.52 x^{1.63}}}{1-e^{-97.52}}-\frac{1-e^{-10.86 y^{1.04}}}{1-e^{-10.86}}-\frac{1-e^{-52.43 z^{0.84}}}{1-e^{-52.43}}
$$

$(x, y, z)$ are positive variables, and each of the elementary cost function has negative exponents. The magnitude of the exponent thus has to be as large as possible to minimize $\Omega(x, y, z)$, and we can expect Constraint (1) to become an equality which leads to $x+y+z=1$. Minimizing $\Omega(x, y, z)$ becomes a 2-dimensional problem where $y$, and $z$ are the two independent variables $(x=1-y-z)$. The goal is to minimize $F(y, z)=\Omega((1-y-z), y, z)$ on the domain that satisfies the constraints presented above. Solving this minimization problem gives similar solutions for both reliability conditions:

$$
\begin{aligned}
& \rightarrow(x, y, z)=(0.232,0.651,0.117) \text { for the Constant High condition, } \\
& \rightarrow(x, y, z)=(0.228,0.654,0.118) \text { for the Constant Low condition. }
\end{aligned}
$$

This solution indicates that, for both reliability conditions, the optimal strategy would be to sample the monitoring, tracking and resource lookzone every $4.3 \mathrm{sec}, 1.5 \mathrm{sec}$ and $8.5 \mathrm{sec}$, respectively. This optimal solution exceeds what operators could achieve since the model assumes constant looking at the MAT battery. In reality, participants look away from the screen fairly regularly to perform control actions on the keyboard. This solution should thus not be regarded as a goal that participants should achieve but rather as a reference against which strategies can be compared. 


\section{APPLICATION TO PRIOR EMPIRICAL DATA}

\subsection{Summary of previous results}

In Bagheri \& Jamieson (2004), 24 participants completed four 30-minute sessions on the MAT battery for a total of 12 10-minute blocks. Automation reliability - defined as the percentage of the 16 malfunctions in each block that were corrected by the automation - was varied as a between-subjects factor. It was either constant at $87.5 \%$ (Constant High), constant at 56.25\% (Constant Low), or changed every block from $87.5 \%$ to $56.25 \%$ (Variable Hi-lo), or from $56.25 \%$ to $87.5 \%$ (Variable Lo-hi).

The effect of reliability on detection rate was shown to be significant $F$ (3, $20)=11.92, p<.001$. Post-hoc analysis revealed that the detection rate of Constant High participants' was poorer than that in any other condition.

\subsection{Attention results}

The sampling rates $(x, y, z)$ were evaluated for each participant to determine the overall cost $\boldsymbol{\Omega}(\boldsymbol{x}, y, z)$ of his/her attention allocation strategy.

Overall cost function. Reliability had a significant effect on the log transformed cost function $F(3,20)=12.29, p<.001$. Constant High participants exhibited a significantly more costly behaviour than those in any other condition (Figure 3). They also had a sampling strategy significantly different from the optimal one $t(45)=4.72, p<.001$. It should however be noted that this was also the case for the Constant Low and the Variable Hi-lo participants, $t(44)=4.02, p<.001, t(50)=3.13, p<.01$, respectively.

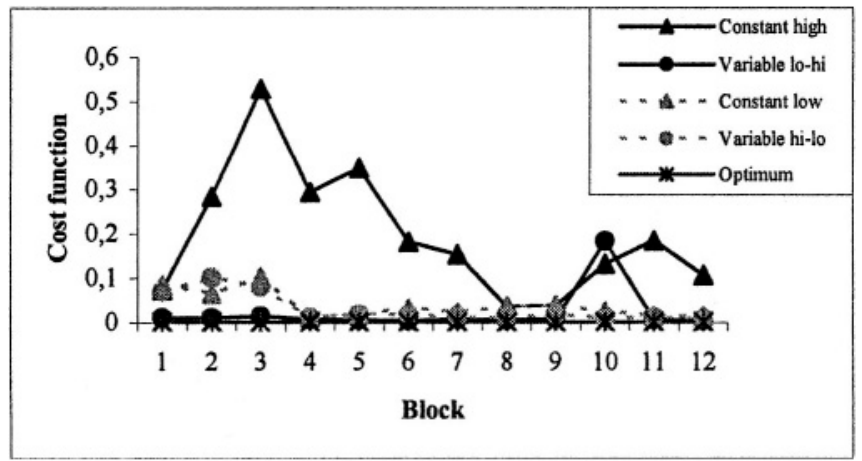

Figure 3. Overall cost functions by reliability group and block 
The effect of block on the overall cost function approached significance $F(11,121)=1.66, p=0.09$ (Figure 3 ). In each condition, participants developed a less costly sampling strategy over time.

Elementary cost functions $C_{m} C_{k} C_{h}$. Reliability had no significant effect on either the tracking or the resource cost function $F(3,20)=1.41, p>.05$, $F(3,20)=0, p>0.05$, respectively. This was confirmed by participants' performance on both tasks, where no effect of reliability was found (see Bagheri \& Jamieson, 2004). However, reliability had a significant effect on the cost function of the monitoring task $F(3,20)=19.50, p<.0001$. The difference observed in the overall cost function thus appears to be due to the sampling strategies for the monitoring task (Figure 4). Constant High participants had a significantly more costly behavior than what could be obtained when optimally sampling this task $t(45)=4.11, p<0.001$, whereas variable participants' behavior did not significantly differ from the optimum.

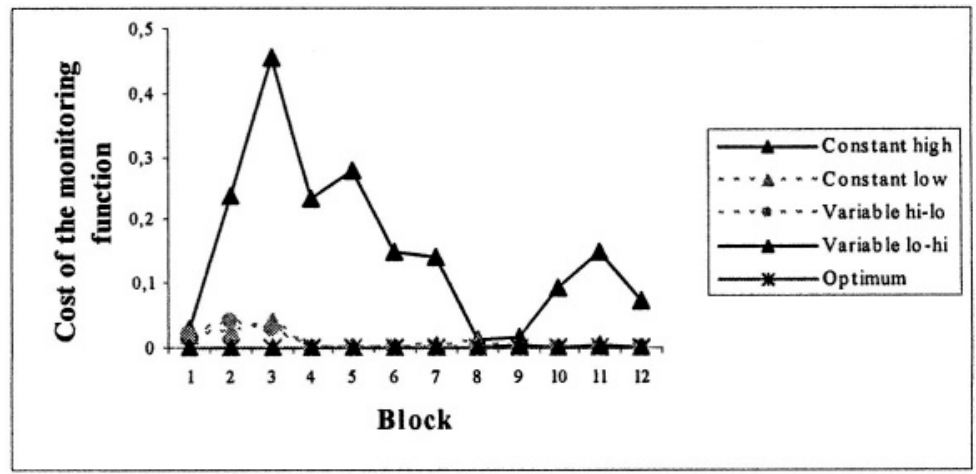

Figure 4. Cost of the monitoring task by reliability and block

\section{DISCUSSION}

The data from Bagheri and Jamieson (2004) showed that automation failure detection was significantly worse for Constant High participants, which could indicate that these participants were complacent. The model we presented here allowed us to consider this possible attribution more closely.

Eye point of gaze data first revealed that all participants (except those in the Variable Lo-hi condition) exhibited an overall strategy significantly more expensive than the optimal one. This difference was related to participants' tendency to sample the tracking lookzone less, and the resource lookzone more, than what was optimal (a trend observed in all conditions). 
Moreover, the attention allocation strategy of Constant High participants was more costly than that of participants in all other conditions.

As shown in Table 5, very similar sampling rates are required for both reliability conditions to achieve a desired detection rate. This led to very similar monitoring cost functions and optimal sampling rates. As a result, Constant High participants' strategies could be compared not only to the optimal strategy but also to that of participants in other conditions. As shown in Figure 4, Constant High participants sampled the monitoring task significantly less than participants in the other reliability conditions, accounting for the higher cost of their overall strategy. The monitoring behavior of these participants could be interpreted as evidence of complacent behavior since better performance could be achieved with higher sampling rates as illustrated both by participants in the other conditions and the optimal solution. However, it is critical to note that after an increase of their monitoring cost function across the first three blocks, Constant High participants started to monitor more efficiently. By Block 8 , they exhibited a monitoring behaviour comparable to that of participants in the other conditions and a trend converging toward the optimal behavior. This dynamic argues against the presence of complacency as complacent behavior is hypothesized to develop with prolonged interaction with highly reliable automation (Prinzel et al., 2001). This change in attention strategy was not observed from detection results (which remained significantly below that of participants in other conditions), which emphasizes the importance of measuring attention when evaluating monitoring performance.

It must be highlighted that a $100 \%$ detection rate of automation failures equates to detecting 7 failures in a block with low reliability automation and only 2 failures in a block with high reliability automation. However, for both reliability conditions, the same sampling rate is required to achieve this detection rate since automation failures occur randomly and unpredictably. Indeed, the MAT battery presents no local context that would allow participants to suspect that a failure might occur. Thus, sampling at a given rate is more rewarding for participants facing low reliable automation, or more 'laborious' for those facing highly reliable automation as they 'look for nothing' more often. It must be noted that the cost of 'looking for nothing' was only included as an indirect cost in the model, the cost of potentially be missing events in the unobserved lookzones. Jerison and Wing (1963) argued that detection rate provides reinforcement and hence control of the rate of the 'observing response'. Thus, detecting a failure could reinforce the sampling of the monitoring task. The design of this task, which requires a similar sampling rate regardless of automation reliability to reach a given detection rate, could thus partly explain the Constant High participants' poorer performance. 
It can be seen that Constraint (1) is not that restrictive. Given the dynamics of the three tasks, participants could perform almost optimally on each of them as illustrated by the values of the cost function associated with the optimal solution: $C_{m}=0.00018, C_{t}=0.00095, C_{r}=0.00017$. If the monitoring task was not automated, sampling the lookzone at the optimal rate previously defined would still allow participants to detect almost all of the malfunctions. This begs the question of the need to automate the task. Indeed, if the sampling rate necessary to detect all the failures is the same regardless of the reliability, then low reliable automation might lead to a more efficient behavior as it would better "reward" participants for looking at the monitoring task, and might prevent complacency from happening. This might even be a better option, since the sampling rate necessary to detect all of the failures does not seem to prevent efficient performance on the other tasks. If this holds across other studies of multi-task performance, it would have implication for the difficult problem of function allocation.

Model limitations. There are several limitations to the presented model. First, the operator was assumed to be a periodic sampler. Although, this might be true for the continuous tracking task, and perhaps the monitoring task, we would expect the operator to sample the resource lookzone more often when the fuel level approaches the boundaries. A model like Carbonell's (1966), in which the sampling interval depends on the distance from the boundary, might have been more appropriate, although much more complex.

A second limitation comes from the small sample used to verify that the equation of the different cost functions was sufficiently close to experimental data. A more accurate approximation could be obtained with more subjects and a wider range of sampling rates.

Third, the influence of control actions on sampling rate was not considered in our model. When control actions are performed, uncertainty due to the action is added to the existing background uncertainty (Crossman et al., 1974). Sampling intervals following control actions tend to decrease until the expected effects of the control changes have worn off.

\section{CONCLUSION}

We have developed a sampling model that allows us to determine the efficiency of a participants' attention allocation strategy. When applied to previously reported empirical data, the model demonstrated that users of constant, highly reliable automation exhibited monitoring behaviour that was more 'expensive' than (1) the optimal strategy and (2) that of participants in other reliability conditions. However, the evolution of their strategy does not 
support the attribution of poor monitoring performance to complacency. This model also casts doubt on the need to automate the monitoring task in the MAT Battery and suggests that the simulation should be modified to more meaningfully assess monitoring of automation in multi-task environments.

\section{ACKNOWLEDGMENT}

Financial support was provided by NSERC. Frederic Winther greatly contributed to the development of the model. Roy Kwon provided valuable assistance in solving the optimization equation.

\section{REFERENCES}

Bagheri, N. \& Jamieson, G. (2004). Considering subjective trust and monitoring behavior in assessing automation-induced "complacency". In Proceeding of the Human Performance, Situation Awareness and Automation Technology II Conference, Daytona Beach, FL.

Carbonell, J. (1969). A queuing model of many-instrument visual sampling. IEEE Transactions on Human Factors in Electronics, HFE-5, 156-164.

Crossman, E., Cooke, J. \& Beishon, R. (1978). Visual attention and displayed information in process control. In Edwards, E. \& Lees, F. The Human Operator in Process Control.

Jerison, H. \& Wing, J. (1963). Human vigilance and operant behavior. In D. Buckner \& J. McGrath (Eds.), Vigilance: a symposium. New York: McGraw Hill.

Moray, N. (2003). Monitoring, complacency, scepticism and eutectic behaviour. International Journal of Industrial Ergonomics, 31,175-178.

Moray, N., T. Inagaki (2000). Attention and complacency. Theoretical Issues in Ergonomics Science, 1, 354-365.

Moray, N. \& Inagaki, T. (1999). Laboratory studies of trust between humans and machines in automated systems. Trans. Inst. MC, 21, No. 4/5, pp. 203-211.

Moray, N. (1986). Monitoring behavior and supervisory control. In Boff, K., Kaufman, L. \& Thomas, J. (Eds), Handbook of perception and human performance.

Parasuraman, R., Molloy, R. \& Singh I. L. (1993). Performance consequences of automationinduced "complacency". The International Journal of Aviation Psychology, 3, 1-23.

Prinzell, L. J., DeVries, H., Freeman, F.G., \& Mikulka, P. (2001). Examination of Automation-Induced Complacency and Individual Difference Variates. (Tech. Memorandum No. 211413). Hampton, VA: NASA Langley Research Center.

Schumacher, W. \& Geiser, G. (1983). Human control strategies in concurrent binary tasks under overload conditions. Automatica, Vol. 19, pp.723-727.

Singh, I., Molloy, R. \& Parasuraman, R. (1997). Automation-induced monitoring efficiency: role of display location. International Journal of Human-Computer Studies, 46, 17-30.

Wiener, E. (1981). Complacency: Is the term useful for air safety? In Proceedings of the $\mathbf{2 6}^{\text {th }}$ Corporate Aviation Safety Seminar, pp. 116-125. Denver: Flight Safety Foundation, Inc. 